\title{
Adaptation to the Interferon-Induced Antiviral State by Human and Simian Immunodeficiency Viruses
}

\author{
Julia Bitzegeio, ${ }^{a, b}$ Marissa Sampias, ${ }^{a}$ Paul D. Bieniasz, ${ }^{a, b, c}$ Theodora Hatziioannou ${ }^{a}$ \\ Aaron Diamond AIDS Research Center, The Rockefeller University, New York, New York, USAa; Laboratory of Retrovirology, The Rockefeller University, New York, New York,

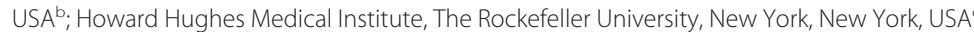

The production of type I interferon (IFN) is an early host response to different infectious agents leading to the induction of hundreds of IFN-stimulated genes (ISGs). The roles of many ISGs in host defense are unknown, but their expression results in the induction of an "antiviral state" that inhibits the replication of many viruses. Here we show that prototype primate lentiviruses human immunodeficiency virus type 1 (HIV-1) and simian immunodeficiency virus of macaques $\left(\right.$ SIV $_{M A C}$ and SIV $\left._{M N E}\right)$ can replicate in lymphocytes from their usual hosts (humans and macaques, respectively), even when an antiviral state is induced by IFN- $\alpha$ treatment. In contrast, HIV-1 and SIV ${ }_{M A C} / S_{\text {MNE }}$ replication was hypersensitive to IFN- $\alpha$ in lymphocytes from unnatural hosts, indicating that the antiviral state can effectively curtail the replication of primate lentiviruses in hosts to which they are not adapted. Most of the members of a panel of naturally occurring HIV-1 and HIV-2 strains behaved like prototype strains and were comparatively insensitive to IFN- $\alpha$ in human lymphocytes. Using chimeric viruses engineered to overcome restriction factors whose antiretroviral specificities vary in a species-dependent manner, we demonstrate that differential $\mathrm{HIV}-1$ and $\mathrm{SIV} \mathrm{MAC}_{\mathrm{C}}$ sensitivities to IFN- $\alpha$ in lymphocytes from humans and macaques could not be ascribed to TRIM5, APOBEC3, tetherin, or SAMHD1. Single-cycle infection experiments indicated that at least part of this species-specific, IFN- $\alpha$-induced restriction of primate lentivirus replication occurs early in the retroviral life cycle. Overall, these studies indicate the existence of undiscovered, IFN- $\alpha$-inducible antiretroviral factors whose spectrum of activity varies in a species-dependent manner and to which at least some HIV/SIV strains have become adapted in their usual hosts.

R ecognition of viruses or other microbes through pathogenassociated molecular patterns leads to the expression of interferons (IFNs) (1) and constitutes one of the earliest host responses to an infection. Thereafter, the binding of type I IFN (IFN- $\alpha / \beta)$ to a receptor (IFNAR) that is present on many cell types activates a JAK-STAT signaling cascade, leading to the expression of hundreds of IFN-stimulated genes (ISGs) and the induction of an antiviral state (2). For both humans and macaques infected with human immunodeficiency virus type 1 (HIV-1) or simian immunodeficiency virus of macaques $\left(\mathrm{SIV}_{\mathrm{MAC}}\right)$, respectively, high levels of IFN- $\alpha$ (up to $>1,000 \mathrm{U} / \mathrm{ml}$ ) have been reported during acute infection (3-6). Moreover, in pathogenic primate lentivirus infections, a chronic activation of the immune system, including induction of ISGs, continues throughout the course of the disease $(4,7)$. Despite the induction of IFN- $\alpha$ expression, HIV-1 and $\mathrm{SIV}_{\mathrm{MAC}}$ replication persists.

Among the many genes induced by IFN- $\alpha$ are those that encode so-called "restriction factors." These proteins have been shown to directly inhibit viral replication through various mechanisms $(8,9)$. To enable replication in the presence of restriction factors, primate lentiviruses have evolved ways to evade or counteract them, sometimes employing accessory genes as restriction factor antagonists. The activity of restriction factors and their antagonism by viral proteins vary in a highly species-dependent manner. Often, primate viruses have evolved to evade or antagonize restriction factors in their usual host species but remain sensitive to variants of the same factors in other hosts $(8,9)$. For example, HIV-1 and HIV-2 have evolved strategies to counteract human tetherin (10-12), while $\mathrm{SIV}_{\text {MAC }}$ is sensitive to inhibition by human tetherin (13). In contrast, $\mathrm{SIV}_{\mathrm{MAC}}$ can efficiently antagonize macaque tetherin but HIV-1 and HIV-2 cannot $(13,14)$. Conceptually similar species-dependent evasion or antagonism phenomena are evident for each of the restriction factors that are known to target primate lentiviruses, namely, APOBEC3, TRIM5, tetherin, and SAMHD1 (8). Importantly, these restriction factors provide barriers to the cross-species transmission of primate lentiviruses and complicate the development of suitable animal models for the study of HIV-1 and other primate lentiviruses (15). Indeed, macaques, which are often used as a model system for HIV-1 infection in humans, express several factors that inhibit HIV-1, but generally not $\mathrm{SIV}_{\mathrm{MAC}}$, infection. Especially potent blocks to HIV-1 replication in macaque cells are imposed by TRIM $5 \alpha$, which inhibits incoming HIV-1 particles by recognizing the HIV-1 capsid (CA) protein, (16) and APOBEC3 proteins that catalyze the deamination of deoxycytidines in viral DNA during reverse transcription (17-19). Whereas the SIV $\mathrm{MAC}_{\mathrm{A}} \mathrm{CA}$ and Vif proteins can evade and antagonize the macaque TRIM5 and APOBEC3 proteins, respectively, the HIV-1 CA and Vif proteins do not.

Overcoming TRIM5 $\alpha$ - and APOBEC3-based restrictions by

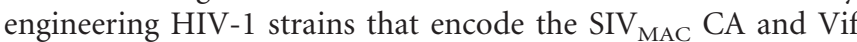
proteins has made it possible to develop a simian-tropic HIV-1 strain (stHIV) that is able to replicate in rhesus macaque lymphocytes (20). Moreover, the discovery that pigtailed macaques express a TRIM5-cyclophilin A fusion protein (TRIMCyp), that is

Received 19 November 2012 Accepted 7 January 2013

Published ahead of print 16 January 2013

Address correspondence to Paul Bieniasz,pbienias@adarc.org, or Theodora

Hatziioannou, thatziio@adarc.org.

Copyright @ 2013, American Society for Microbiology. All Rights Reserved.

doi:10.1128/JVI.03219-12 
not active against HIV-1 (21-23) enabled the construction of an stHIV strain in which the only alteration was the inclusion of SIV $_{\text {MAC }}$ Vif (24). This stHIV strain replicates efficiently in pigtailed macaque lymphocytes in vitro. However, although viremia is robust during the acute infection phase in vivo, it progressively declines over time and no disease is evident (24), suggesting the possibility that additional species-dependent restrictions to primate lentiviruses exist that limit stHIV replication in vivo.

Here we have examined whether the IFN- $\alpha$-induced host response imposes a host species-dependent block to primate lentivirus replication. We found that this is indeed the case and that stHIV and two prototype SIVs are hypersensitive to inhibition by IFN- $\alpha$ when asked to replicate in primary lymphocytes from unnatural hosts. We demonstrated that these blocks are not attributable to TRIM5, APOBEC3, tetherin, or SAMHD1 and are manifested, at least in part, during the early stages of the virus life cycle. Moreover, naturally occurring HIV-1 and HIV-2 strains are generally less sensitive to IFN- $\alpha$ than the prototype SIVs in human lymphocytes are. Overall, these studies indicate the existence of novel IFN- $\alpha$-inducible factors whose antiretroviral activity varies in a manner that is dependent on the particular primate lentivirus and the host cell species.

\section{MATERIALS AND METHODS}

Plasmid construction. The proviral plasmids stHIV and stHIV(SCA), which are based on the HIV-1 clone NL4-3, have been described previously $(20,24)$. In stHIV, the Vif coding sequences have been replaced with

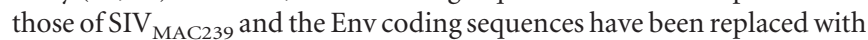
those of the macaque-adapted HIV-1 envelope from SHIV/KB9. In stHIV(SCA), the HIV-1 CA-encoding sequences have also been replaced with those of $\operatorname{SIV}_{\text {MAC239. }}$

The proviral plasmid stHIV $\Delta$ Vpu was generated by using overlap extension PCR to replace the first two codons of the stHIV Vpu codon with six nucleotides encoding a BamHI site (GGATCC). For the construction of stHIVdenU and stHIVgsnU, overlap extension PCR was used to replace stHIV Vpu coding sequences with those from SIVdenVpu or SIVgsnVpu. Because the Vpu and Env sequences overlap, this manipulation resulted in the replacement of the KB9 Env amino terminus with that of SIVden Env or SIVgsn Env. However, the Vpu-Env overlap is confined to the Env signal peptide, so the amino acid sequence of mature, functional KB9 Env was unaltered (see Results).

The proviral plasmid stHIV $\Delta$ envGFP has been described previously (20). Derivatives thereof (stHIV $\Delta$ vpu $\Delta$ envGFP, stHIVdenU $\Delta$ envGFP, and stHIVgsnU $\Delta$ envGFP) were generated by combining fragments of stHIV $\Delta$ envGFP with stHIV $\Delta$ Vpu stHIVdenU or stHIVgsnU.

To generate stHIVVpx, overlap extension PCR was used to insert SIV $_{\text {MAC239 }}$ Vif and Vpx sequences, rather than Vif alone, between the $3^{\prime}$ end of HIV-1 pol and the 5' end of HIV-1 vpr, as was described for the original generation of stHIV $(20,24)$ (see Results). A proviral plasmid (stHIVSp6) encoding the Vpx packaging signal from SIV $_{\text {MAC239 }}$ p6 was generated essentially as previously described (25). The stHIVSp6Vpx proviral plasmid (see Fig. 5A) was generated by combining fragments of stHIVSp6 and stHIVVpx.

A plasmid containing the full-length $\operatorname{SIV}_{\mathrm{MAC} 239}$ proviral DNA was generated on the basis of a pXf3-derived low-copy-number vector termed V1 and using p239SpSp5' and p239SpE3' obtained from the NIH AIDS Research and Reference Reagent Program and contributed by R. C. Desrosiers, J. S. Gibbs, and D. Regier (26). A $\operatorname{SIV}_{\text {MAC239 }} \Delta$ Nef proviral plasmid was similarly derived. For the generation of $\operatorname{SIV}_{\mathrm{MAC} 239} \Delta \mathrm{Vpx}$, the Vpx initiation codon was mutated without changing the amino acid sequence in the overlapping Vif protein. The $\mathrm{SIV}_{\mathrm{MNE} 027}$ proviral plasmid has been described elsewhere (27).

A proviral plasmid, SIV-GFP, encoding green fluorescent protein
(GFP) in place of $\operatorname{SIV}_{\mathrm{MAC} 239} \mathrm{Nef}$ has been described previously (28). To obtain stHIV-GFP and stHIV(SCA)-GFP, an stHIV fragment containing $\mathrm{SIV}_{\text {MAC }}$ Vif was introduced into HIV/KB9-GFP and HIV(SCA)KB9-GFP, respectively, which have been described previously (20).

The plasmid coding for hemagglutinin (HA)-tagged human tetherin has been described previously (29). A similar plasmid encoding pigtailed HA-tagged macaque tetherin (13) was constructed by using similar approaches. PCR primer sequences used for the generation of the above constructs can be obtained upon request. The plasmid encoding rhAPOBEC3G has been previously described (19).

Proviral plasmids encoding transmitted founder HIV-1 strains were obtained through the AIDS Research and Reference Reagent Program, Division of AIDS, NIAID, NIH (Panel of Infectious Molecular Clones; catalog number 11919), from John Kappes (30-34).

Cell culture. The adherent cell lines $293 \mathrm{~T}$ and TZM were maintained in Dulbecco's modified Eagle's medium supplemented with $10 \%$ fetal calf serum (FCS) and gentamicin. CEMx174 suspension cells were grown in RPMI medium with 10\% FCS and gentamicin. Human peripheral blood mononuclear cells (huPBMCs) were isolated from blood by Ficoll-Paque gradient centrifugation. Isolated cells were activated with phytohemagglutinin (PHA-P; Sigma) and 5\% interleukin-2 (IL-2; Hemagen) for $48 \mathrm{~h}$ and then grown in the presence of 5\% IL-2 in RPMI medium supplemented with $10 \%$ FCS. Frozen stocks of pigtailed macaque PBMCs (pgtPBMCs) were kindly provided by Jeff Lifson. After thawing, cells were activated with $3 \mu \mathrm{g} / \mathrm{ml}$ staphylococcal enterotoxin B and 5\% IL-2 for $48 \mathrm{~h}$ and then maintained in RPMI medium with 10\% FCS and 5\% IL-2.

Transfection and virus production. 293T cells were seeded at $2.5 \times$ $10^{5} /$ well (24-well plate) or $6 \times 10^{6} / 10-\mathrm{cm}$ dish and transfected the following day using polyethylenimine (PolySciences). For the generation of virus stocks, $293 \mathrm{~T}$ cells in a $10-\mathrm{cm}$ plate were transfected with $15 \mu \mathrm{g}$ of proviral plasmids and placed in fresh medium after $24 \mathrm{~h}$. At $40 \mathrm{~h}$ posttransfection, virus-containing cell supernatant was harvested, clarified by low-speed centrifugation, filtered $(0.22 \mu \mathrm{m})$, and stored at $-80^{\circ} \mathrm{C}$. To generate vesicular stomatitis virus glycoprotein G (VSV-G)-pseudotyped virus stocks, $1 \mu \mathrm{g}$ of a VSV-G expression plasmid was added to the transfection mixture. To compare the activities of Vif proteins against rhAPOBEC3G, a myc-tagged APOBEC3G plasmid (19) was cotransfected with full-length viruses and at $48 \mathrm{~h}$ posttransfection, cell lysates were harvested and analyzed by immunoblotting.

To determine infectious virus titers, serial dilutions of the virus stock were used to infect TZM reporter cells seeded into 96-well plates at a density of $3 \times 10^{3} /$ well. At $48 \mathrm{~h}$ postinfection, the cells were fixed with $0.5 \%$ glutaraldehyde and stained with 5 -bromo-4-chloro-3-indolyl- $\beta$-Dgalactopyranoside (X-Gal) to determine the number of infected foci. Titers of GFP reporter viruses were determined by infecting CEMx174 cells with serial dilutions of the stock. At $48 \mathrm{~h}$ postinfection, the number of infected cells was determined by fluorescence-activated cell sorting (FACS).

Cell-free HIV-2 stocks were obtained through the AIDS Research and Reference Reagent Program, Division of AIDS, NIAID, NIH (HIV-2 7294A, HIV-2 7312A, and HIV-2 60415K from Feng Gao and Beatrice Hahn and HIV-2 CDC310072, HIV-2 CDC310319, and HIV-2 CDC77618 from Stefan Wiktor and Mark Rayfield) $(35,36)$. HIV-2 stocks were generated by short-term culture (to avoid any potential in vitro adaptation) in huPBMCs.

rhAPOBEC3G antagonism. To determine activity against

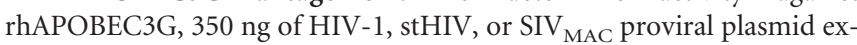
pressing GFP in place of Nef was cotransfected with $350,175,87.5$, or $43.75 \mathrm{ng}$ of an rhAPOBEC3G expression plasmid or a control empty vector. The total amount of DNA was held constant by supplementing the transfection with empty expression vector. At $48 \mathrm{~h}$ posttransfection, supernatants were harvested, clarified by low-speed centrifugation, and filtered $(0.22 \mu \mathrm{m})$ and titers were determined by infecting CEMx174 cells.

Western blot analyses. Cell lysates and virions pelleted through $20 \%$ sucrose $\left(20,000 \times g\right.$ for $2 \mathrm{~h}$ at $\left.4^{\circ} \mathrm{C}\right)$ were separated on NuPage Novex 4 to 
12\% Bis-Tris Mini Gels (Invitrogen). Proteins were blotted onto nitrocellulose membranes. Thereafter, blots were blocked in blocking solution (Rockland) and probed with the primary antibody and a corresponding IRDye $800 \mathrm{CW}$ - or IRDye 680-conjugated secondary antibody. Fluorescent signals were detected and quantified with an Odyssey scanner (LI-COR Biosciences). HIV-1 CA was detected with the mouse monoclonal antibody 183-H12-5C (37) from Bruce Chesebro and Hardy Chen. SIV $_{\text {MAC239 }}$ Vpx was detected with a Vpx monoclonal antibody (6D2.6) (38) from John C. Kappes. HIV-1 gp41 was detected with monoclonal antibody F240 (39) from Marshall Posner and Lisa Cavacini, and tetherin was detected with a polyclonal rabbit serum (40) from Klaus Strebel and Amy Andrew. Each of the aforementioned antibodies was acquired through the NIH AIDS Research and Reference Reagent Program.

Virus replication assay. Freshly activated PBMCs were infected with HIV-1, stHIV, HIV-2, or SIV strains at a multiplicity of infection (MOI) of 0.001 (infectious titers were determined on TZM cells). Alternatively, viral stocks were normalized for reverse transcriptase (RT) activity prior to infection. At $16 \mathrm{~h}$ postinfection, cells were washed and divided into two

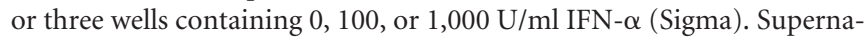
tants were collected every $48 \mathrm{~h}$ for the following 13 to 15 days. The amount of virus particles released into the cell culture supernatant was determined by measuring RT activity (Lenti RT Activity kit; Cavidi).

Virus release assays. Proviral plasmids $(500 \mu \mathrm{g})$ were cotransfected into 293T cells with increasing amounts of plasmids expressing human or pigtailed macaque tetherin $(0 \mathrm{ng}, 11 \mathrm{ng}, 33 \mathrm{ng}$, and $100 \mu \mathrm{g})$. At $48 \mathrm{~h}$ posttransfection, virion-containing supernatants were harvested, clarified by low-speed centrifugation, and filtered. Infectious virus release was determined by inoculating TZM indicator cells, which had been plated the previous day in 96 -well plates at $8 \times 10^{3}$ cells/well, with $50 \mu$ l of serially diluted supernatants. At $48 \mathrm{~h}$ after infection, $\beta$-galactosidase activity was determined with GalactoStar reagent (PerkinElmer). The remainder of the virion-containing supernatant $(750 \mu \mathrm{l})$ was layered onto $400 \mu \mathrm{l}$ of $20 \%$ sucrose in phosphate-buffered saline and centrifuged at 20,000 $\times g$ for $2 \mathrm{~h}$ at $4^{\circ} \mathrm{C}$. Virion pellets and corresponding virion-producing cells were dissolved in SDS-PAGE loading buffer, and Western blot analysis was performed as described above.

Single-cycle PBMC infection assays. Freshly activated huPBMCs or pgtPBMCs seeded at a density of $5 \times 10^{4} /$ well of a 96 -well plate were

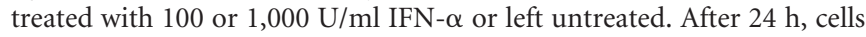
were infected with GFP-expressing reporter viruses. After a further $24 \mathrm{~h}$, the medium was changed and $100 \mathrm{ng} / \mathrm{ml}$ lamivudine (3TC) was added to prevent further virus spreading. At $48 \mathrm{~h}$ postinfection, the cells were fixed and the percentage of GFP-positive cells was determined by FACS.

CD4 downregulation assay. CEMx174 cells were infected with VSVG-pseudotyped stHIV $\Delta e n v$ GFP reporter viruses expressing the various $\mathrm{V}$ pu proteins. The cells were stained at $48 \mathrm{~h}$ postinfection with a mouse anti-human CD4 antibody (RPA-T4) conjugated to Alexa 700 (BD Pharmingen) and fixed in paraformaldehyde. Cell-associated fluorescence in the 700-nm and GFP channels was measured with an LSRII flow cytometer (BD), and data were analyzed with FlowJo software.

\section{RESULTS}

Host cell species-dependent effects of IFN- $\alpha$ on HIV/SIV replication. To test the hypothesis that unidentified ISGs might inhibit primate lentivirus replication in unnatural-host cells, we compared the replication of $\mathrm{SIV}_{\mathrm{MAC239}}, \mathrm{SIV}_{\mathrm{MNE027}}$, and stHIV in huPBMCs and pgtPBMCs in the presence or absence of IFN- $\alpha$. The stHIV strain used was based on NL4-3 but encoded a dual-tropic envelope protein (from SHIV/KB9), as well as the $\mathrm{SIV}_{\text {MAC239 }}$ Vif protein (24) (Fig. 1A). In contrast to HIV-1, expressing HIV-1 Vif, stHIV antagonized rhAPOBEC3G as efficiently as $\operatorname{SIV}_{\text {MAC }}$ (Fig. 1B), suggesting that both viruses express SIV $_{\text {MAC }}$ Vif at approximately equivalent levels. As ex-

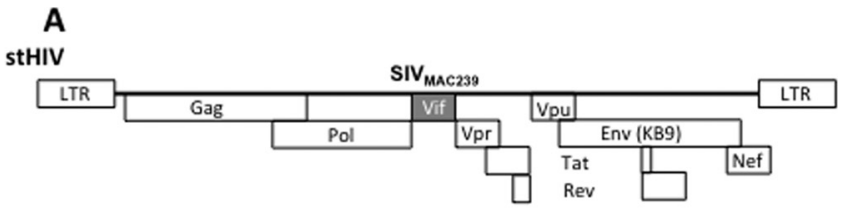

B

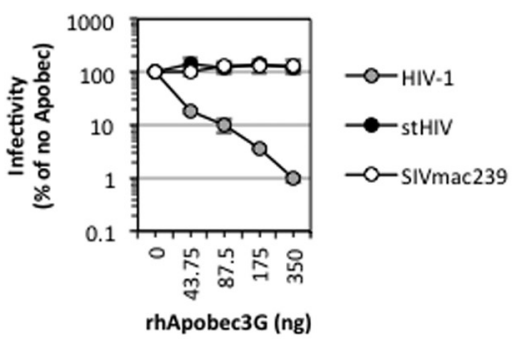

$\multimap$ no IfN $\rightarrow-100 \mathrm{U} / \mathrm{ml}$ IFN $\rightarrow-1000 \mathrm{U} / \mathrm{ml}$ IFN

C

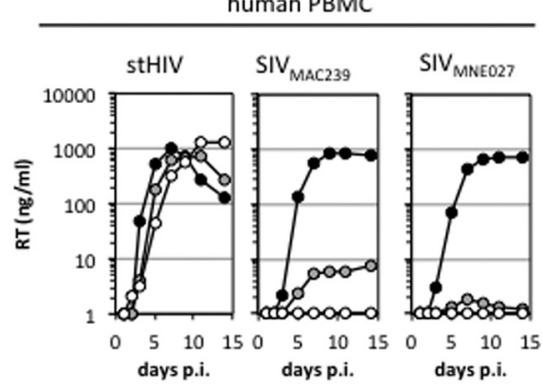

D

pigtailed macaque PBMC

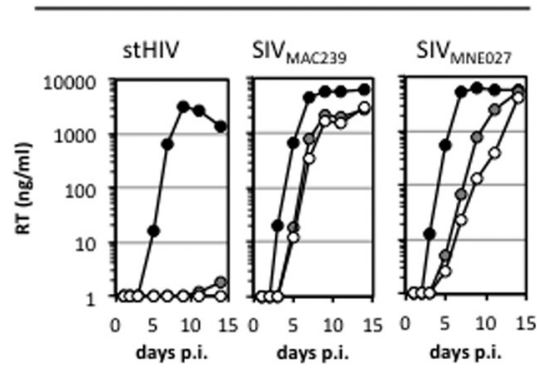

FIG 1 Differential sensitivities of stHIV and SIV $_{\text {MAC239 }}$ to IFN- $\alpha$ in huPBMCs and pgtPBMCs. (A) Schematic representation of the stHIV genome. The Vif protein is from $\mathrm{SIV}_{\mathrm{MAC} 239}$, and the Env protein is from SHIV/KB9. All other sequences are from NL4-3. (B) Equivalent amounts of HIV-1, stHIV, and SIV $_{\text {MAC }}$ proviruses expressing GFP were cotransfected with various amounts of rhAPOBEC3G. Supernatants were collected at $48 \mathrm{~h}$ posttransfection and assayed for infectivity on CEM cells. (C and D) Activated huPBMCs (B) or pgtPBMCs (C) were infected with stHIV or $\mathrm{SIV}_{\text {MAC239 }} / \mathrm{SIV}_{\mathrm{MNE}}$ at an MOI of 0.001 . At $24 \mathrm{~h}$ postinfection, the cells were washed and samples were split into three wells, two of which were treated with 100 or $1,000 \mathrm{U} / \mathrm{ml}$ IFN- $\alpha$. Supernatants were collected every $48 \mathrm{~h}$ for the following 15 days, and RT activity was measured with an enzyme-linked immunosorbent assay (Cavidi Tech). LTR, long terminal repeat; p.i., postinfection.

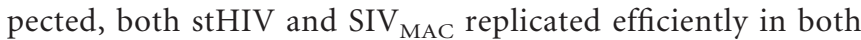
huPBMCs and pgtPBMCs (Fig. 1C and D). However, addition of IFN- $\alpha$ completely suppressed the replication of $\mathrm{SIV}_{\text {MAC239 }}$

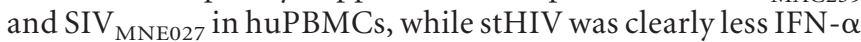
sensitive (Fig. 1C). Conversely, in pgtPBMCs, IFN- $\alpha$ completely suppressed stHIV replication while $\mathrm{SIV}_{\mathrm{MAC239}}$ and $\mathrm{SIV}_{\text {MNE027 }}$ were less sensitive (Fig. 1D). Of note, the doses of 
A

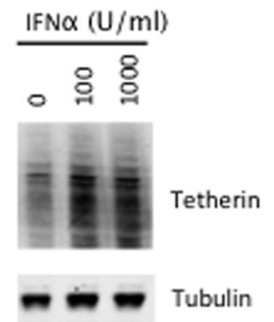

B sthivdenU

stHIVgsnU

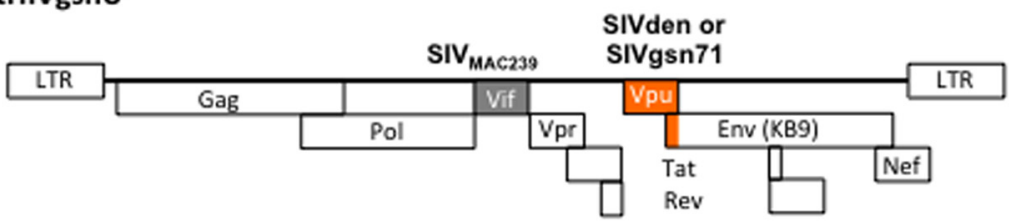

\footnotetext{
Signal peptidase cleavage

$\downarrow$

stHIVdenU Env:

MSOVRRTLKVLLLILWTIGIICTEKLWVTVYY

stHIVgsnU Env: MMRKVGFLKMLVVSLTLMALLPIPGSRSKGTEKLWVTVYY
}

C

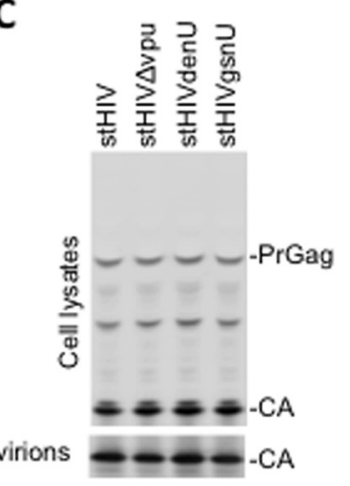

D

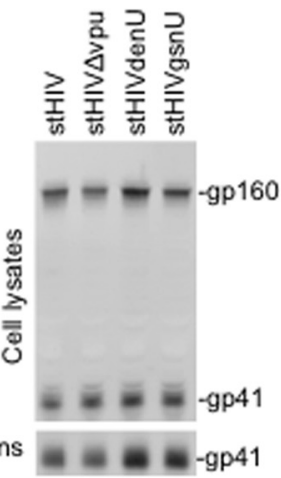

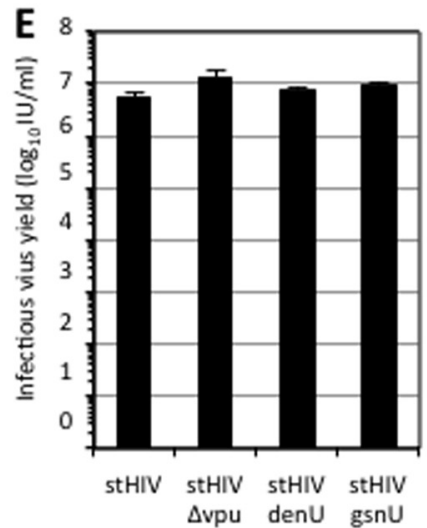

stHIV $\Delta \mathrm{Vpu}$

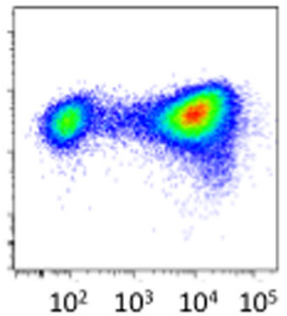

stHIVdenU

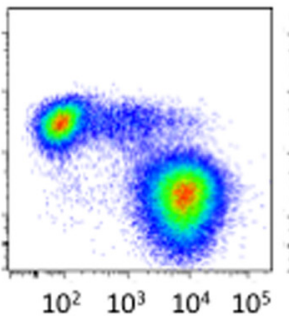

stHIVgsnU

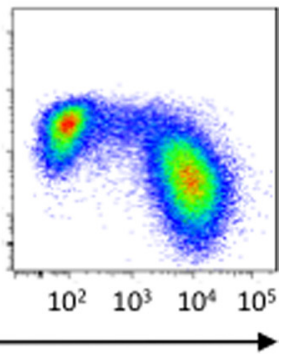

GFP

FIG 2 Functional replacement of the stHIV Vpu protein with SIVden Vpu or SIVgsn Vpu. (A) huPBMCs left untreated or treated with 100 or 1,000 U/ml IFN- $\alpha$ for $24 \mathrm{~h}$ were lysed and subjected to Western blot analysis with antitetherin and antitubulin antibodies. Note that tetherin is highly and heterogeneously glycosylated and migrates as a smear at 50 to $64 \mathrm{kDa}$. (B) Schematic representation of the stHIVdenU and stHIVgsnU genomes. The Vpu proteins and Env signal peptides are from SIVden or SIVgsn. All other sequences are from the same stHIV construct described in Fig. 1A. (C) and (D) 293T cells were transfected with stHIV, stHIV $\Delta \mathrm{vpu}$, stHIVdenU, or stHIVgsnU. At $48 \mathrm{~h}$ posttransfection, the virions were harvested, cells were lysed, and both were subjected to Western blot analysis with anti-CA (B) or anti-gp41 (C) antibodies. (E) Virion-containing supernatants were titrated on TZM reporter cells, and infected foci were revealed by X-Gal staining and counted. (F) CEMx174 cells were infected with stHIVDenvGFP reporter viruses pseudotyped with VSV-G containing the gene for GFP in the place of the gene for Nef. At $48 \mathrm{~h}$ postinfection, the cells were fixed and stained for CD4 and analyzed by flow cytometry. LTR, long terminal repeat. 
A

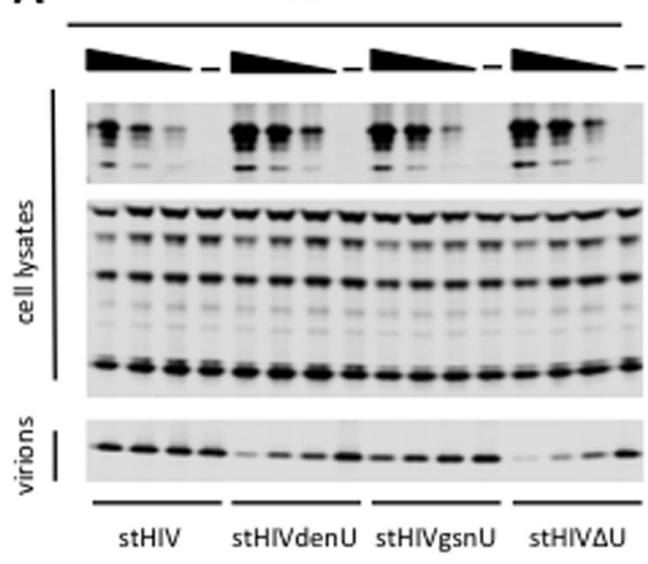

B

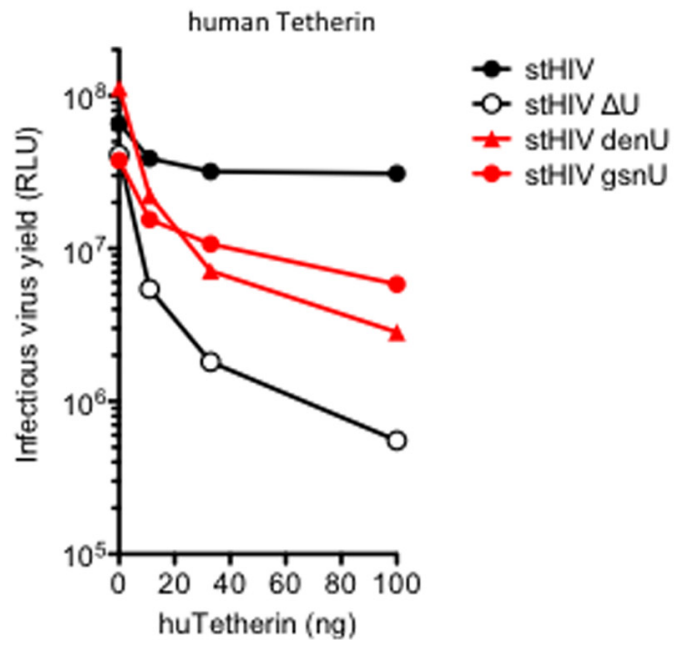

Pigtailed macaque Tetherin
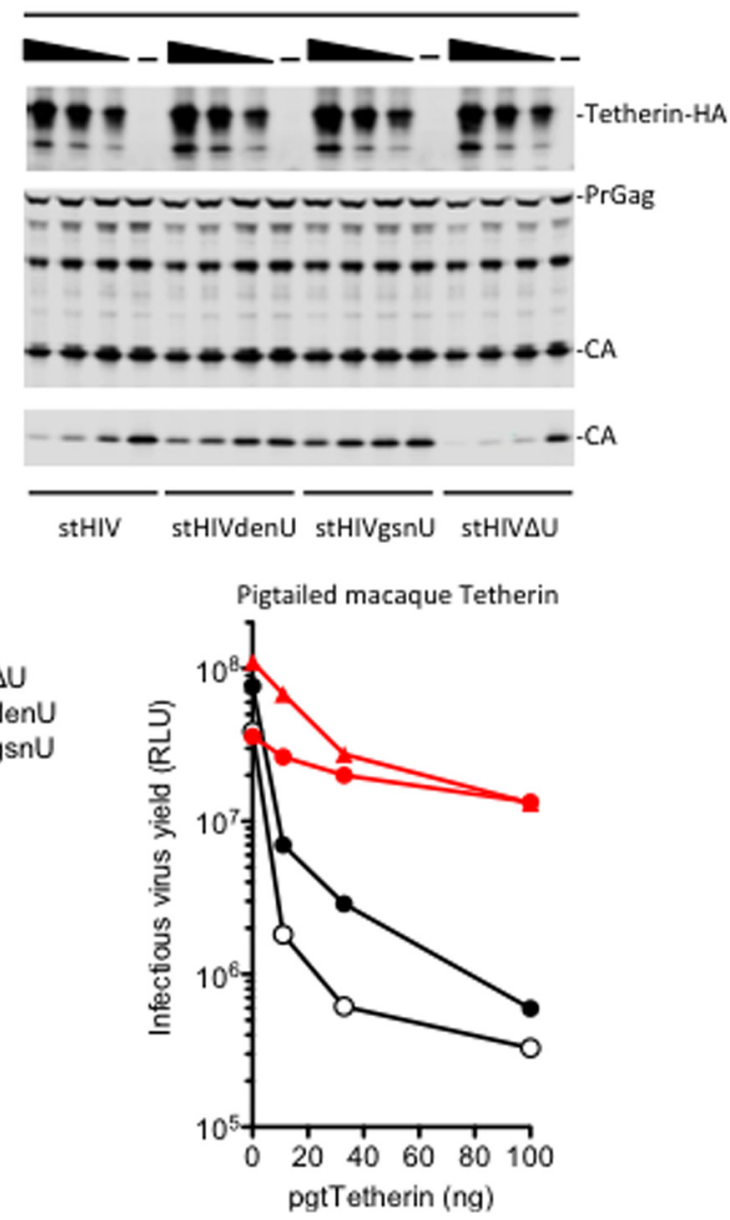

FIG 3 Resistance of stHIVdenU and stHIVgsnU to pigtailed macaque tetherin. (A) Western blot analysis of 293T cells cotransfected with the indicated proviral plasmid and increasing amounts $(0,11,33$, or $100 \mathrm{ng})$ of a plasmid expressing either human or pigtailed macaque tetherin. Cell and virion lysates were probed with anti-CA monoclonal and/or rabbit anti-HA antibodies, and signals were detected with fluorescent secondary antibodies. (B) Virion-containing supernatants harvested at $48 \mathrm{~h}$ postinfection were titrated on TZM reporter cells, and the infectious virion yield was determined with a chemiluminescent $\beta$-galactosidase assay. RLU, relative light units.

IFN- $\alpha$ ( 100 or $1,000 \mathrm{U} / \mathrm{ml})$ used in Fig. 1 are within the range of those induced by HIV-1 or SIV $\mathrm{MAC} 239_{9}$ replication in the respective hosts (3-6).

These data suggest that one or more IFN- $\alpha$-induced antiretroviral activities are expressed in the normal target cells of primate lentiviruses and specifically inhibit viruses that are not adapted to replicate in that particular species. Notably, such antiretroviral activities are unlikely to reflect the activity of TRIM5 proteins since neither HIV-1 nor $\operatorname{SIV}_{\mathrm{MAC2} 39}$ is sensitive to inhibition by the human or pigtailed macaque forms of these factors (41). Moreover, since stHIV and SIV $_{\text {MAC239 }}$ encode identical Vif proteins, these species-specific effects of IFN- $\alpha$ cannot be ascribed to the APOBEC 3 proteins that are targeted by Vif and are known to be active against primate lentiviruses (Fig. 1B).

Tetherin does not account for species-dependent effects of IFN- $\boldsymbol{\alpha}$ on HIV/SIV replication. Another antiviral protein that could, in principle, be responsible for the virus- and host-specific inhibition of primate lentiviruses by IFN- $\alpha$ is tetherin. stHIV encodes an HIV-1 Vpu protein that is unable to antagonize tetherin variants in most nonhuman primates, including macaques (14,
29). Because tetherin is induced severalfold in PBMCs following IFN- $\alpha$ treatment (11) (Fig. 2A), it might have been responsible for the IFN- $\alpha$-induced inhibition of stHIV replication in pgtPBMCs.

We recently identified Vpu proteins from SIV strains that are effective antagonists of macaque tetherin proteins (42), and so we used these to replace the HIV-1 Vpu protein in stHIV. Specifically, we generated two stHIV variants, namely, stHIVdenU and stHIVgsnU, that express the Vpu proteins from SIVden (43) and SIVgsn71 (44), respectively (Fig. 2B). We also generated an stHIV variant that does not express a Vpu protein (stHIV $\Delta \mathrm{U})$. When 293T cells were transfected with stHIV, stHIV $\Delta$ U, stHIVdenU, or stHIVgsnU proviral plasmids, they expressed viral Gag proteins and generated extracellular virions at levels comparable to those obtained with stHIV (Fig. 2C). Because the $3^{\prime}$ portion of the Vpu sequence overlaps the Env signal peptide sequence, both stHIVdenU and stHIVgsnU contain signal peptides that are not native to HIV-1 (Fig. 2B). However, these are cleaved upon Env maturation and were not predicted to affect the expression or function of stHIV Env. Indeed, Env expression and incorporation into particles were similar for stHIV, stHIV $\Delta \mathrm{U}$, stHIVdenU, and 

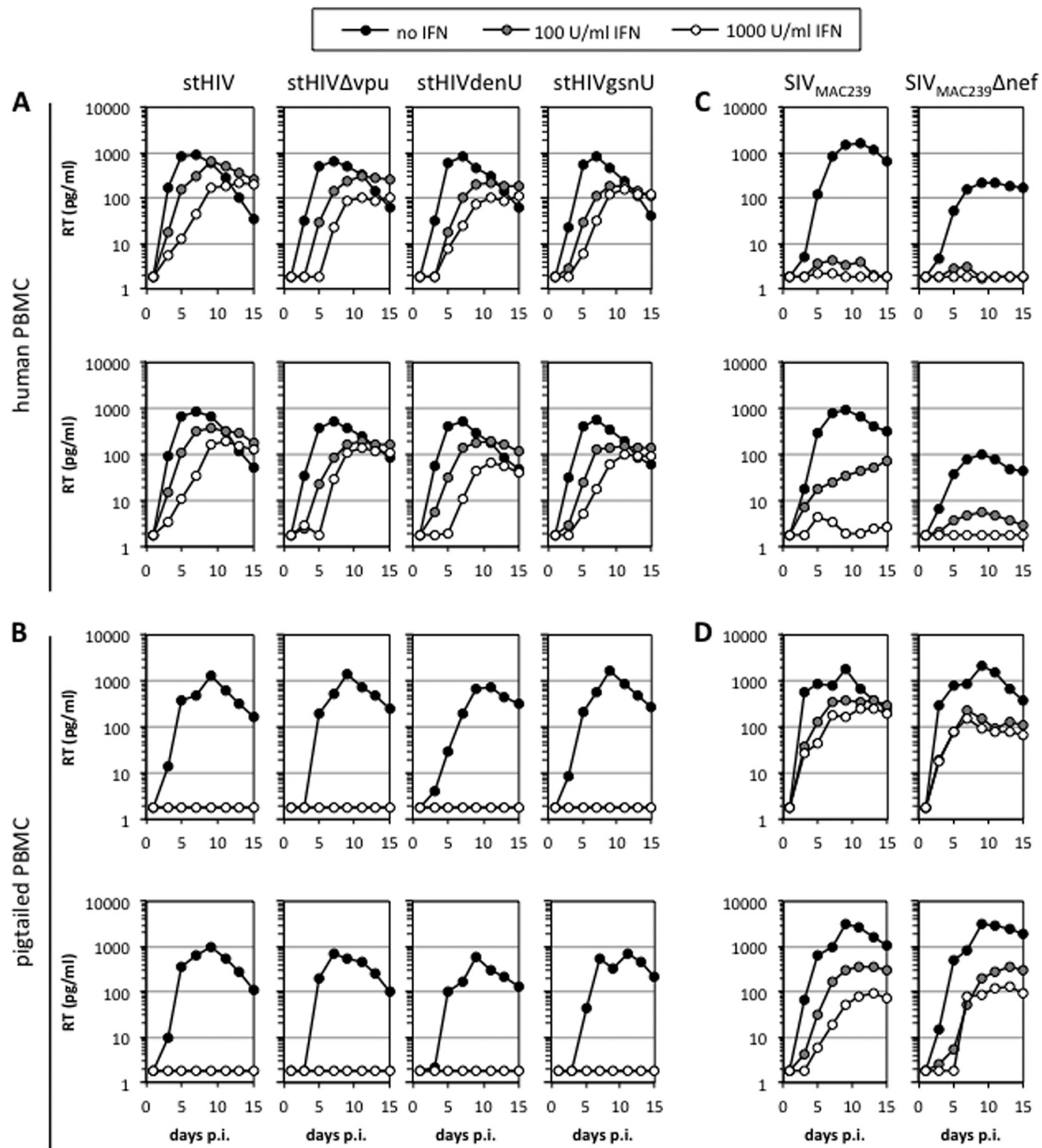

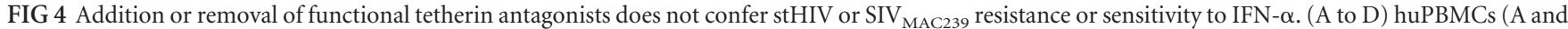
C) or pgtPBMCs (B and D) were infected with stHIV, stHIV $\Delta \mathrm{vpu}$, stHIVdenU, stHIVgsnU (A and B), SIV $\mathrm{MAC239}_{\text {, or SIV }} \mathrm{MAC} 239_{2} \Delta \mathrm{Nef}_{(\mathrm{C}}$ and D) at an MOI of 0.001 . The next day, cells were washed and divided among three wells that were treated with 0,100 , or $1,000 \mathrm{U} / \mathrm{ml}$ IFN- $\alpha$. Supernatants were collected every 48 $\mathrm{h}$ for the following 15 days, and RT activity was measured with an enzyme-linked immunosorbent assay-based assay (Cavidi Tech). Results from two huPBMC and pgtPBMC donors are shown. Note that the virus replication curves in the presence of 100 or $1,000 \mathrm{U} / \mathrm{ml}$ IFN- $\alpha$ (gray and open circles) are superimposed in panel B. p.i., postinfection.

stHIVgsnU (Fig. 2D). Additionally, each construct yielded particles similar in infectiousness (Fig. 2E).

To verify that the various Vpu proteins were expressed in a functional form, we generated Env-defective derivatives of stHIV, stHIVdenU, stHIVgsnU, and stHIV $\Delta \mathrm{U}$ that expressed GFP in place of Nef. In these constructs, the only viral protein that is capable of inducing CD4 downregulation is Vpu. Infection of CEMx174 cells with VSV-G-pseudotyped virions generated with each of the constructs (with the exception of stHIV $\Delta \mathrm{U}$ ) induced efficient CD4 downregulation (Fig. 2F). Finally, to confirm that each Vpu protein possessed tetherin antagonist activity when expressed in the context of stHIV, we cotransfected the panel of
stHIV constructs with increasing amounts of plasmids expressing either human tetherin or pigtailed macaque tetherin and measured particle release by Western blot (Fig. 3A) and infectivity (Fig. 3B) assays. As expected, stHIV, which expresses HIV-1 Vpu, was largely resistant to human tetherin but sensitive to pigtailed macaque tetherin, while stHIV $\Delta \mathrm{U}$ was sensitive to both tetherin proteins (Fig. 3A and B). In contrast, stHIVdenU and stHIVgsnU were largely resistant to pigtailed macaque tetherin but displayed intermediate sensitivity to human tetherin (Fig. 3A and B). Importantly, because stHIVdenU and stHIVgsnU were significantly less sensitive than stHIV to pigtailed macaque tetherin, these experiments established reagents that could test whether the host 

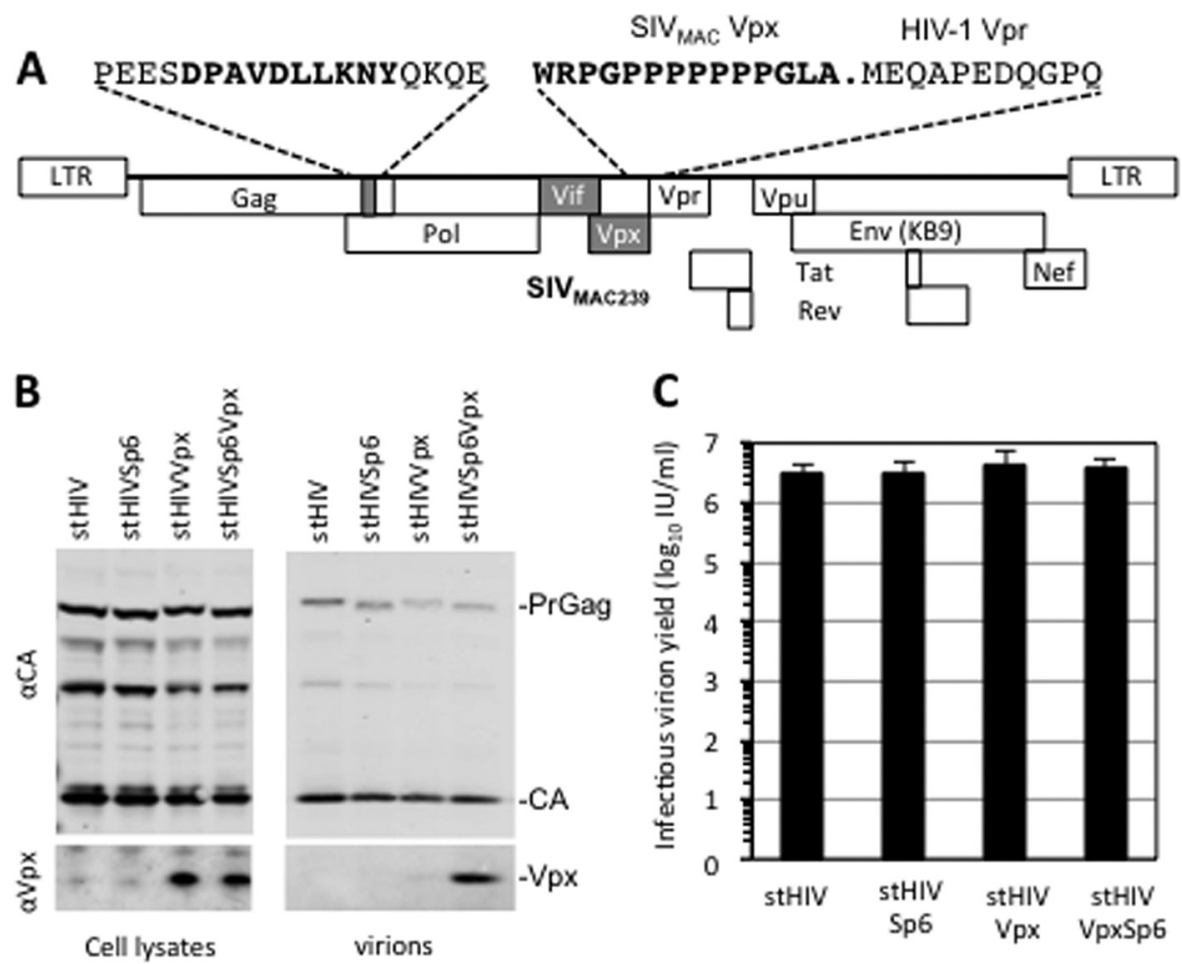

FIG 5 Insertion of SIV ${ }_{\text {MAC239 }}$ Vpx into the stHIV genome and incorporation into infectious virions. (A) Schematic representation of the stHIVSp6Vpx genome. The Vpx proteins and the Vpx packaging signal within $\mathrm{p} 6$ are from $\mathrm{SIV}_{\mathrm{MAC} 239}$. All other sequences are from the stHIV construct described in Fig. 1A. (B) Western blot analysis of 293T cells and virions transfected with stHIV, stHIVSp6, stHIVVpx, and stHIVSp6Vpx that were harvested at $48 \mathrm{~h}$ postinfection. Cell lysates and virions were probed with anti-CA and anti-Vpx monoclonal antibodies, and signals were detected with fluorescent secondary antibodies. (C) Virion-containing supernatants were titrated on TZM reporter cells, and infected foci were revealed by X-Gal staining and counted. LTR, long terminal repeat.

cell species-dependent effects of IFN- $\alpha$ on stHIV replication could be attributed to tetherin. Specifically, if tetherin is the major effector of the IFN- $\alpha$-induced block to stHIV replication, then stHIVdenU and stHIVgsnU should be less sensitive than stHIV and stHIV $\Delta U$ to IFN- $\alpha$ in pgtPBMCs and more sensitive than stHIV to IFN- $\alpha$ in huPBMCs. As a complementary approach, we also used an $\operatorname{SIV}_{\text {MAC239 }} \Delta$ Nef construct. Nef is the only SIV MAC239 $_{2}$ protein that can antagonize tetherin $(13,14)$. Therefore, $\mathrm{SIV}_{\mathrm{MAC2} 29} \Delta$ Nef should be more sensitive to IFN- $\alpha$ in pgtPBMCs if tetherin is the sole (or major) effector of IFN- $\alpha$-induced antiretroviral activity therein.

In the absence of IFN- $\alpha$, all of the aforementioned viruses replicated to comparable levels in huPBMCs and pgtPBMCs (Fig. 4A and $\mathrm{B}$ ). In the presence of $100 \mathrm{U} / \mathrm{ml}$ or $1,000 \mathrm{U} / \mathrm{ml}$ IFN- $\alpha$, the stHIV $\Delta \mathrm{Vpu}$, stHIVdenU, and stHIVgsnU variants replicated well in huPBMCs, but a modest delay in replication was evident compared to stHIV (compare day 3 and day 5 RT values in Fig. 4A; data not shown). This delay was most likely due to the inability of these variants to efficiently counteract human tetherin, which, as previously reported, is upregulated in huPBMCs in the presence of IFN- $\alpha$ (11) (Fig. 2A). Importantly, stHIV, stHIV $\Delta \mathrm{Vpu}$, stHIVdenU, and stHIVgsnU were equally sensitive to IFN- $\alpha$ in pgtPBMCs, which abolished replication therein (Fig. 4B). These findings indicate that IFN- $\alpha$-induced inhibitors other than tetherin are the major factors limiting stHIV replication in IFN- $\alpha$ treated pgtPBMCs. In support of this conclusion, $\mathrm{SIV}_{\mathrm{MAC} 239} \Delta \mathrm{Nef}$ did not exhibit increased sensitivity to IFN- $\alpha$ in pgtPBMCs, while both wild-type $\mathrm{SIV}_{\mathrm{MAC} 239}$ and $\mathrm{SIV}_{\mathrm{MAC} 239} \Delta \mathrm{Nef}$ were very sensitive to IFN- $\alpha$ in huPBMCs (Fig. $4 \mathrm{C}$ and D).
SAMHD1 does not account for species-dependent effects of IFN- $\boldsymbol{\alpha}$ on HIV/SIV replication. One major difference between

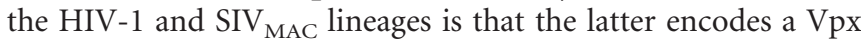
protein that is known to counteract SAMHD1 $(45,46)$. SAMHD1 expression is modestly induced by IFN- $\alpha$ in some cell types (P.D.B., unpublished observations) and is a deoxynucleoside triphosphate triphosphohydrolase (47) that reduces the levels of intracellular deoxynucleoside triphosphates below the concentration needed for efficient reverse transcription (48). Vpx proteins, delivered to target cells as a component of virions, can interact with SAMHD1 and target it for proteasomal degradation, thereby alleviating restriction $(45,46)$. Although $\operatorname{SIV}_{\mathrm{MAC}} \mathrm{Vpx}$ is able to counteract both human and macaque SAMHD1 $(49,50)$, it was possible that quantitative differences in the efficiency of Vpx-mediated antagonism of human versus macaque SAMHD1 might

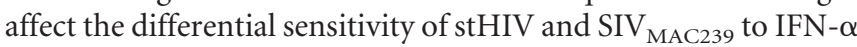
in pgtPBMCs. Alternatively, it was possible that Vpx might have some other function that enabled replication in IFN- $\alpha$-treated pgtPBMCs but was unnecessary in huPBMCs.

The HIV-1 p6 domain of Gag is unable to recruit SIV $_{\text {MAC239 }}$ $\mathrm{Vpx}$ into particles $(51,52)$. However, introduction of a Vpx pack-

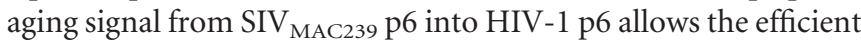
incorporation of $\mathrm{SIV}_{\mathrm{MAC} 239} \mathrm{Vpx}$ into HIV-1 particles (25). On the basis of this finding, we generated three stHIV-based proviral plasmids that included the minimal $\mathrm{SIV}_{\text {MAC239 }} \mathrm{Vpx}$ packaging signal within p6 (25) (stHIVSp6), the $\operatorname{SIV}_{\text {MAC239 }}$ Vpx protein (stHIVVpx), or both (stHIVSp6Vpx) (Fig. 5A). For each construct, Gag expression and particle release upon the transfection of 293T cells were comparable to those of stHIV (Fig. 5B). Fur- 

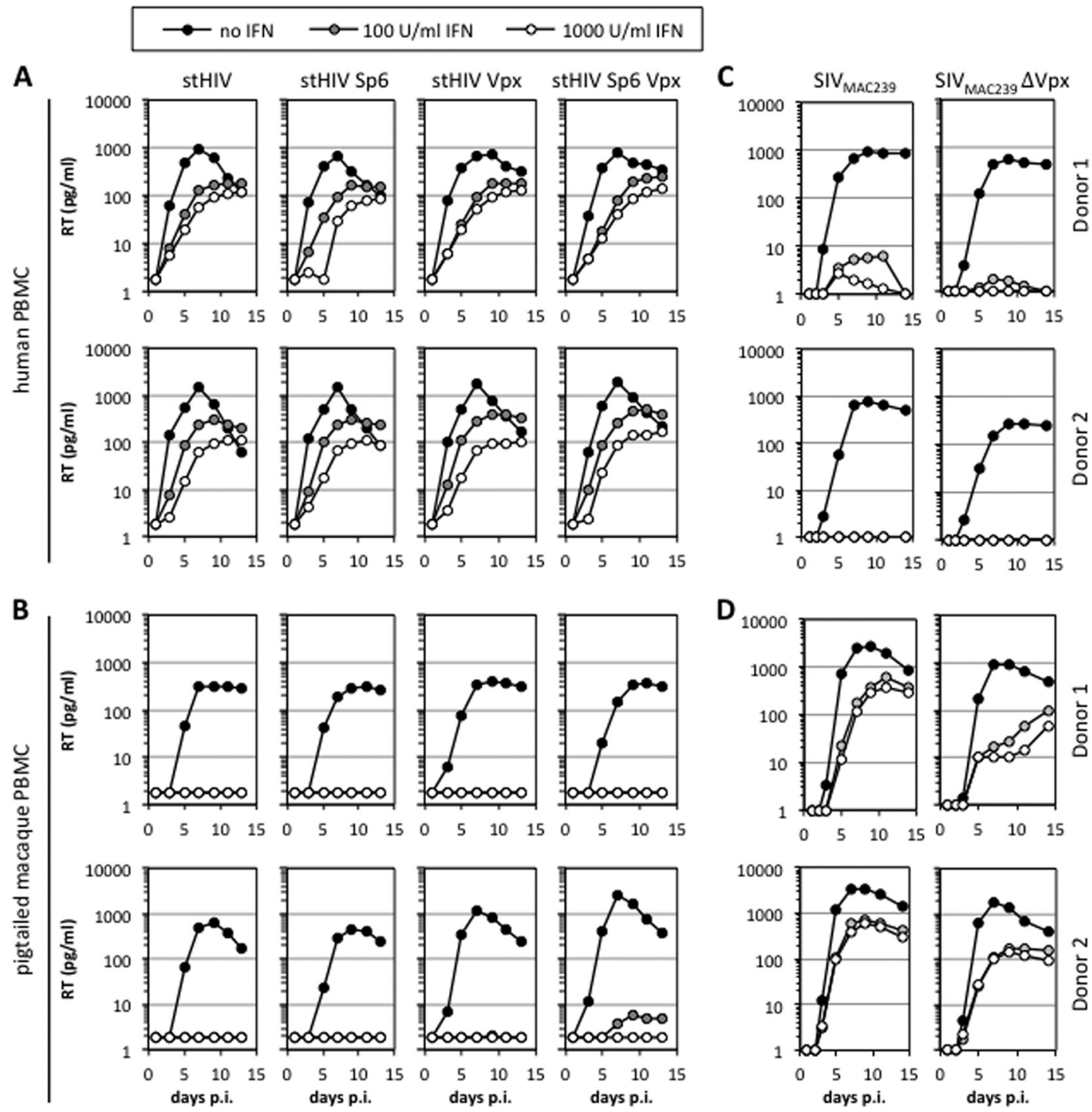

FIG 6 Insertion of Vpx into or its removal from the stHIV or SIV $_{\text {MAC239 }}$ genome does not confer resistance or sensitivity to IFN- $\alpha$. (A to D) huPBMCs (A and C) or pgtPBMCs (B and D) were infected at an MOI of 0.001 with stHIV, stHIVSp6, stHIVVpx, or stHIVSp6Vpx (A and B) or with SIV MAC239 $_{\text {or SIV }}$ MAC239 $\Delta$ Vpx $(\mathrm{C}$ and $\mathrm{D})$. The next day, cells were washed and divided among three wells that were treated with 0,100 , or $1,000 \mathrm{U} / \mathrm{ml}$ IFN- $\alpha$. Supernatants were collected every $48 \mathrm{~h}$ for the following 15 days, and RT activity was measured with an enzyme-linked immunosorbent assay-based assay (Cavidi Tech). Results from two huPBMC and pgtPBMC donors are shown. Note that the virus replication curves in the presence of 100 or $1,000 \mathrm{U} / \mathrm{ml}$ of IFN- $\alpha$ (gray and open circles) are superimposed in panels B and C (bottom). p.i., postinfection.

thermore, all of the constructs yielded similar titers of infectious virions using TZM indicator cells (Fig. 5C). As expected, although both stHIVVpx and stHIVSp6Vpx expressed Vpx, only stHIVSp6Vpx was able to package Vpx into particles (Fig. 5B).

Notably, stHIV, stHIVSp6, stHIVVpx, and stHIVSp6Vpx all replicated to similar levels in huPBMCs and exhibited a similar relative lack of sensitivity to IFN- $\alpha$ (Fig. 6A). Moreover, each of these viruses replicated to similar levels in pgtPBMCs and, importantly, replication was completely suppressed in the presence of IFN- $\alpha$ (Fig. 6B). These results indicate that the putative IFN- $\alpha-$ induced inhibitors of HIV-1 replication in pgtPBMCs are not antagonized by SIV $_{\text {MAC239 }} \mathrm{Vpx}$. To corroborate these findings, the replication of $\operatorname{SIV}_{\mathrm{MAC2} 39}$ and $\operatorname{SIV}_{\mathrm{MAC2} 39} \Delta \mathrm{Vpx}$ was analyzed in huPBMCs and pgtPBMCs. In the absence of IFN- $\alpha$, the replication of $\operatorname{SIV}_{\text {MAC239 }} \Delta$ Vpx was marginally reduced in both huPBMCs and
pgtPBMCs compared to that of $\operatorname{SIV}_{\mathrm{MAC239}}$ (Fig. 6C and D). However, $\operatorname{SIV}_{\mathrm{MAC23}} \Delta \mathrm{Vpx}$ was able to replicate in IFN- $\alpha$-treated pgtPBMCs but both viruses were profoundly inhibited by IFN- $\alpha$ treatment in huPBMCs (Fig. 6C and D). Thus, for both stHIV and $\mathrm{SIV}_{\mathrm{MAC239}}$, the addition of $\mathrm{Vpx}$ to or its removal from the viral genome did not affect the host species-dependent IFN- $\alpha$-induced restriction. These results suggest that neither SAMHD1 nor any other potential Vpx target protein is responsible for the IFN- $\alpha$ induced restriction of HIV-1 and SIV $_{\text {MAC239 }}$ replication in unnatural host cells.

IFN- $\alpha$ induces an early, species-dependent but TRIM5-independent block in HIV/SIV infection. The above data strongly suggested that one or more undiscovered IFN- $\alpha$-induced factors are responsible for a species-specific restriction of lentiviral replication. Restriction factors have been shown to act at various stages 


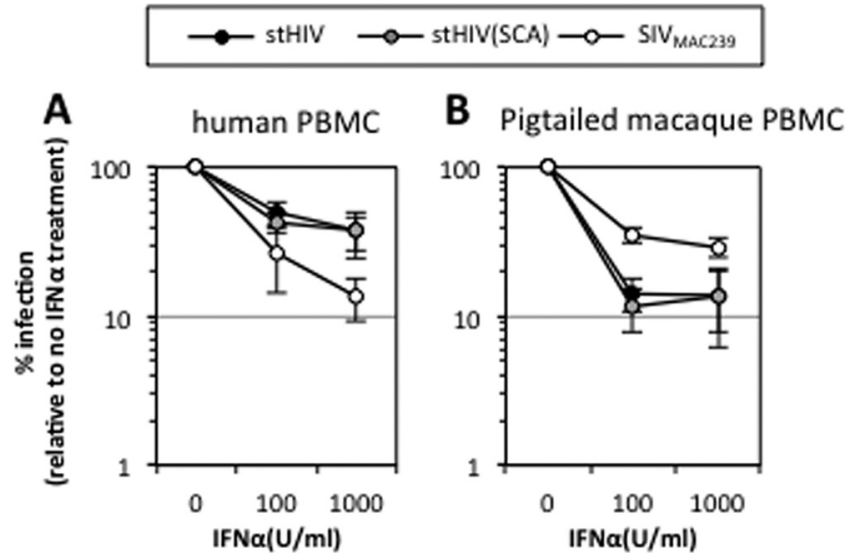

FIG 7 An early, species-dependent but TRIM5-independent block to stHIV and $\operatorname{SIV}_{\text {MAC239 }}$ infection is induced by IFN- $\alpha$. (A and B) huPBMCs (A) or pgtPBMCs (B) were treated with the indicated concentrations of IFN- $\alpha$ for 24 $\mathrm{h}$ and then infected with SIV-GFP, stHIV-GFP, or stHIV(SCA)-GFP reporter virus in the presence of IFN- $\alpha$. At $24 \mathrm{~h}$ postinfection, the medium was changed and $100 \mathrm{ng} / \mathrm{ml}$ 3TC was added. At $48 \mathrm{~h}$ postinfection, the cells were fixed and the percentage of GFP-positive cells was determined by flow cytometry. The data represent the mean \pm standard deviation of three huPBMC or pgtPBMC donors and are plotted relative to the level of infection in the absence of IFN- $\alpha$ (which was set at $100 \%$ ).

of the lentiviral life cycle, so to gain some insight into where such novel factors might act, we conducted single-cycle infection assays

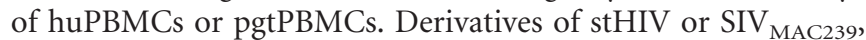
each harboring the GFP protein in the place of Nef were used to quantify infected cells, and to limit the assay to a single cycle of infection, an RT inhibitor (3TC) was added at $16 \mathrm{~h}$ postinoculation.

In this assay format, IFN- $\alpha$ treatment of huPBMCs target cells inhibited single-cycle $\mathrm{SIV}_{\mathrm{MAC} 239} / \mathrm{GFP}$ infectivity in a dose-dependent manner up to $\sim 8$ - to 10 -fold (Fig. $7 \mathrm{~A}$ ). The same treatment inhibited single-cycle stHIV/GFP infectivity by only $\sim 2$ - to 3 -fold. In contrast, IFN- $\alpha$ treatment of pgtPBMC targets inhibited single-cycle $\mathrm{SIV}_{\mathrm{MAC} 239} / \mathrm{GFP}$ infectivity by $\sim 3$-fold but inhibited single-cycle stHIV/GFP infectivity by $\sim 8$ to 10 -fold (Fig. $7 \mathrm{~B}$ ). The greater potency with which IFN- $\alpha$ inhibited single-cycle $\mathrm{SIV}_{\mathrm{MAC239}} / \mathrm{GFP}$ and stHIV/GFP infectivity in PBMCs from unnatural host species was concordant with the effects in spreading replication assays and suggests that at least part of IFN- $\alpha$ 's antiretroviral effect is mediated by a factor(s) inhibiting an early event(s) in the lentiviral life cycle, namely, entry, uncoating, reverse transcription, integration, and/or gene expression.

While the TRIM5 proteins found in humans and pigtailed macaques do not restrict either HIV-1 or $\operatorname{SIV}_{\text {MAC239 }}$ (41), the finding that at least part of the species-dependent effects of IFN- $\alpha$ occurred early in the life cycle prompted us to examine further whether this or any other CA-targeting restriction factor might contribute to the aforementioned effects. To this end, we examined whether an stHIV strain in which the HIV-1 CA domain of Gag was replaced with that of $\operatorname{SIV}_{\text {MAC239 }}[\operatorname{stHIV(SCA)]~(20)~}$ would behave like stHIV or $\mathrm{SIV}_{\mathrm{MAC} 239}$. In fact, during single-cycle infection experiments in which huPBMCs or pgtPBMCs were treated with IFN, stHIV(SCA) displayed the same degree of sensitivity or resistance to inhibition by IFN- $\alpha$ as did stHIV, irrespective of the species from which the PBMCs were derived (Fig. 7A and B). Thus, this experiment effectively excludes TRIM5 proteins and any other CA-targeting factors as mediators of the target cell species-dependent inhibitory activity of IFN- $\alpha$.

Effects of IFN- $\alpha$ on naturally occurring HIV-1 and HIV-2 strains. A caveat of these studies is that stHIV is an engineered derivative of a laboratory-adapted HIV-1 strain (NL4-3). It was necessary to use stHIV in these studies because unmanipulated HIV-1 strains cannot replicate in pgtPBMCs because of blocks imposed by APOBEC3 proteins (24). Nevertheless, it was possible to compare the sensitivity of stHIV to that of more authentic viral strains in huPBMCs. Therefore, we analyzed the effect of IFN- $\alpha$ on the replication of a panel of transmitted founder HIV-1 strains (30-34) in huPBMCs. These naturally occurring HIV-1 strains replicated to levels similar to that of stHIV in untreated huPBMCs (Fig. 8A). Moreover, although there was some PBMC donor-todonor variation, natural HIV-1 strains were clearly more resistant to IFN- $\alpha$ in huPBMCs than were SIV strains (compare Fig. $8 \mathrm{~A}$ with Fig. 1, 4A, and 6A).

Given that $\mathrm{SIV}_{\mathrm{MAC} 239}$ and $\mathrm{SIV}_{\mathrm{MNE} 027}$ were both hypersensitive to IFN- $\alpha$ in huPBMCs (Fig. 1), we also tested whether HIV-2 strains shared this property. HIV-2s are derived from the same lineage as $\mathrm{SIV}_{\text {MAC239 }}$ and $\mathrm{SIV}_{\text {MNE027 }}$, namely, the $\mathrm{SIV}_{\mathrm{SM}}$ group of viruses found in sooty mangabeys (53), but HIV-2 strains have obviously had a greater opportunity to adapt to replication in human lymphocytes and perhaps might be comparatively IFN- $\alpha$ resistant therein. Therefore, we determined the sensitivities of a panel of six HIV-2 strains $(35,36)$ to IFN- $\alpha$ in huPBMCs. In contrast to $\mathrm{SIV}_{\mathrm{MAC} 239}$, primary HIV-2 strains appeared relatively resistant to IFN- $\alpha$ in huPBMCs (Fig. $8 \mathrm{~B}$ ), although two primary HIV-2 strains (HIV-2 ${ }_{7312 \mathrm{~A}}$ and HIV-2 ${ }_{310072}$ ) appeared sensitive, particularly in the presence of higher IFN- $\alpha$ concentrations $(1,000$ $\mathrm{U} / \mathrm{ml}$ ) and depending on the PBMC donor (Fig. 8B; data not shown). Thus, there appeared to be significant differences in IFN- $\alpha$ sensitivity among primary HIV-2 strains that might indicate that certain HIV-2 strains are less well adapted to the IFN- $\alpha$ induced antiviral state in huPBMCs than is HIV-1.

\section{DISCUSSION}

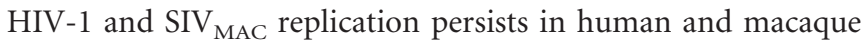
hosts despite a chronic state of immune activation that includes the expression of ISGs and a presumptive "antiviral state" (7). This finding suggests that primate lentiviruses have adapted to replicate in the presence of proteins that might ordinarily inhibit their replication. Indeed, there are clear examples, namely, the APOBEC3 proteins, TRIM5, tetherin, and SAMHD1, where adaptation of primate lentiviruses to the presence of antiretroviral ISGs is demonstrable $(8,9)$.

Nevertheless, previous work has shown that HIV-1 replication can be inhibited in human cells, to at least some degree, by IFN- $\alpha$ $(9,54)$. Indeed, consistent with these observations, we found that

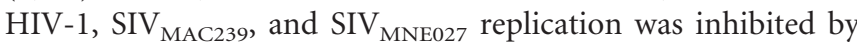
IFN- $\alpha$. However, the degree to which replication was inhibited by IFN- $\alpha$ in PBMCs from their usual hosts was comparatively modest, while each of these viruses was hypersensitive to IFN- $\alpha$ in unnatural host PBMCs. These findings strongly suggest that HIV-1, SIV MAC239, and SIV $_{\text {MNE027 }}$ have adapted, at least to some extent, to replicate in the face of the antiviral state in cells from their usual hosts but not in cells from species outside their usual host range. Although this conclusion held for the most part for HIV-2, we found that two of the six HIV-2 strains tested were 

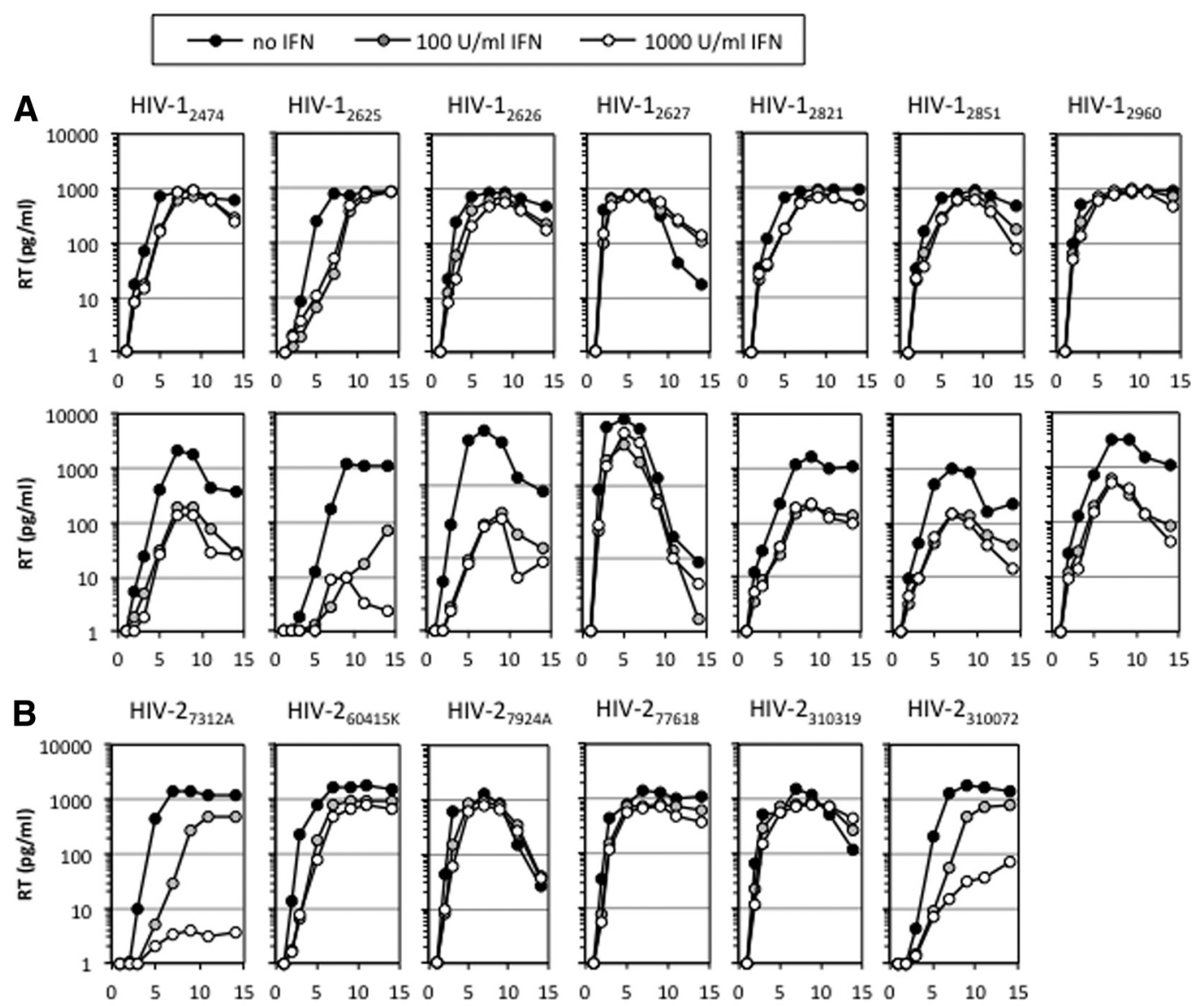

HIV-2 37618

HIV-2 310319

HIV- $2_{310072}$
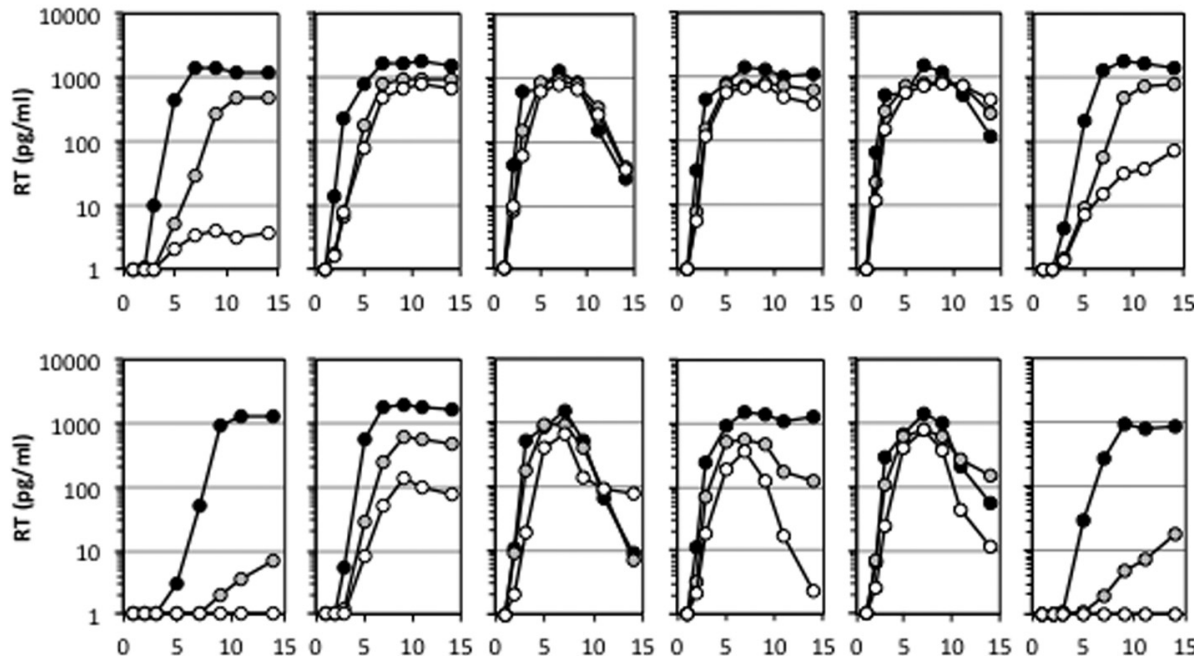

FIG 8 Effects of IFN- $\alpha$ on primary HIV-1 and HIV-2 strain replication in huPBMCs. (A and B) huPBMCs were infected with the indicated HIV-1 (A) or HIV-2 (B) strains at an MOI of 0.001 . The next day, cells were washed and divided among three wells that were treated with 0,100 , or $1,000 \mathrm{U} / \mathrm{ml}$ IFN- $\alpha$. Supernatants were collected every $48 \mathrm{~h}$ for the following 15 days, and RT activity was measured with an enzyme-linked immunosorbent assay (Cavidi Tech). Results from two huPBMC donors are shown.

quite sensitive to IFN- $\alpha$ in huPBMCs. Although the number of strains we analyzed was relatively small, these findings demonstrate strain-to-strain variability in IFN- $\alpha$ sensitivity among HIV-2 strains. Whether this reflects differences among HIV-2 strains in passage history in humans is unclear at present, but it will be interesting to determine IFN- $\alpha$ sensitivity in larger numbers of HIV-2 strains. It is also possible that IFN- $\alpha$ sensitivity varies among HIV-1 and SIV strains, although there is, as yet, no evidence that this is the case.

By using a variety of chimeric viruses encoding nonnative CA, Vif, Vpu, and Vpx proteins, we could demonstrate that IFN- $\alpha$ hypersensitivity in unnatural host cells was unlikely to be attributable to the known restriction factors that target, or are antagonized by, these viral proteins. Indeed, stHIV expresses SIV $_{\text {MAC239 }}$ Vif, effectively excluding the range of APOBEC3 proteins that are targeted by SIV $_{\text {MAC239 }}$ Vif (APOBEC3B, -C, -D, -F, -G, and -H) (19) as mediators of the differential sensitivity of stHIV and $\operatorname{SIV}_{\mathrm{MAC239}}$ to IFN- $\alpha$. Additionally, endowing stHIV with Vpu proteins that could antagonize macaque tetherin or removing the tetherin antagonist (Nef) from SIV $\mathrm{MAC239}_{2}$ did not dramatically alter their sensitivity or resistance to IFN- $\alpha$ in PBMCs from their usual host or those from an unnatural host. In a similar approach, the addition of a $\mathrm{SIV}_{\mathrm{MAC} 239} \mathrm{Vpx}$ protein to stHIV or the removal of $\mathrm{Vpx}$ from $\mathrm{SIV}_{\mathrm{MAC23}}$ did not alter their sensitivity or resistance to IFN- $\alpha$ in usual or unnatural host PBMCs, effectively excluding SAMHD1 as the relevant antiviral protein. Indeed, analysis of HIV-1 replication in cells from Aicardi-Goutières syndrome patients has indicated that the inhibitory effects of SAMHD1 are not evident in activated lymphocytes (55). Moreover, $\mathrm{SIV}_{\mathrm{MAC} 239}$ encodes a Vpx protein 
that can antagonize human SAMHD1 $(45,48)$, while HIV-1 does not, yet $\mathrm{SIV}_{\mathrm{MAC} 239}$ was more sensitive to IFN- $\alpha$ in huPBMCs than was HIV-1.

In the case of TRIM5 proteins, neither huTRIM5 $\alpha$ nor pgtTRIMCyp is active against HIV-1 or $\operatorname{SIV}_{\text {MAC }}(41,56)$. Furthermore, by using single-cycle infectivity experiments, we could demonstrate that the relative resistance of IFN- $\alpha$-treated huPBMCs to $\mathrm{SIV}_{\mathrm{MAC239}}$ and the relative resistance of IFN- $\alpha$-treated pgtPBMCs to stHIV were not determined by the CA protein. Again this excludes TRIM $5 \alpha$ as the determinant of the differential susceptibility of IFN- $\alpha$-treated PBMCs to infection with stHIV and SIV $_{\text {MAC239. }}$

These findings lead to the conclusion that at least one undiscovered ISG is present in cells of macaque origin that can inhibit HIV-1 replication therein. They also suggest that at least one undiscovered ISG is present in huPBMCs that can inhibit SIV replication. Although it is possible that a single polymorphic and relatively potent antiretroviral factor is responsible for all of the species-dependent antiretroviral activities described herein, there is as yet no evidence that this is the case. It is equally possible that several, perhaps many, factors, each with modest antiretroviral activity, contribute to the overall activity of IFN- $\alpha$. Indeed, overexpression screens of hundreds of ISGs for activity against several viruses reveal numerous factors with modest antiviral activities that are additive when combinations of ISGs are coexpressed (57). The identification of such factors will aid both our understanding of how hosts defend against lentivirus infections and the development of HIV-1-based strains that can replicate in unnatural hosts.

\section{ACKNOWLEDGMENTS}

We thank Jeffrey D. Lifson, Gregory Del Prete, and colleagues for providing pgtPBMCs. We are grateful to Frank Kirchhoff for the SIVden and SIVgsn Vpu plasmids. The following reagents were obtained through the AIDS Research and Reference Reagent Program, Division of AIDS, NIAID, NIH: p239SpSp5' and p239SpE3' from R. C. Desrosiers, J. S. Gibbs, and D. Regier; a panel of infectious HIV-1 molecular clones (catalog number 11919) from John Kappes; HIV-2 stocks HIV-2 7294A, HIV-2 7312A, and HIV-2 60415K from Feng Gao and Beatrice Hahn; and HIV-2 CDC310072, HIV-2 CDC310319, and HIV-2 CDC77618 from Stefan Wiktor and Mark Rayfield. We also thank Vineet KewalRamani and members of the Bieniasz and Hatziioannou labs for helpful discussions.

J.B. is funded by a DFG research fellowship (BI 1540/1-1), P.D.B. is funded by the National Institutes of Health (grant R37AI64003) and the Howard Hughes Medical Institute, and T.H. is funded by the National Institutes of Health (grants R01AI078788 and R21AI093255).

\section{REFERENCES}

1. Bowie AG, Unterholzner L. 2008. Viral evasion and subversion of pattern-recognition receptor signalling. Nat. Rev. Immunol. 8:911-922.

2. Der SD, Zhou A, Williams BR, Silverman RH. 1998. Identification of genes differentially regulated by interferon alpha, beta, or gamma using oligonucleotide arrays. Proc. Natl. Acad. Sci. U. S. A. 95:15623-15628.

3. Gaines H, von Sydow MA, von Stedingk LV, Biberfeld G, Bottiger B, Hansson LO, Lundbergh P, Sonnerborg AB, Wasserman J, Strannegaard OO. 1990. Immunological changes in primary HIV-1 infection. AIDS 4:995-999.

4. Jacquelin B, Mayau V, Targat B, Liovat AS, Kunkel D, Petitjean G, Dillies MA, Roques P, Butor C, Silvestri G, Giavedoni LD, Lebon P, Barre-Sinoussi F, Benecke A, Muller-Trutwin MC. 2009. Nonpathogenic SIV infection of African green monkeys induces a strong but rapidly controlled type I IFN response. J. Clin. Invest. 119:3544-3555.

5. Malleret B, Maneglier B, Karlsson I, Lebon P, Nascimbeni M, Perie L, Brochard P, Delache B, Calvo J, Andrieu T, Spreux-Varoquaux O, Hosmalin A, Le Grand R, Vaslin B. 2008. Primary infection with simian immunodeficiency virus: plasmacytoid dendritic cell homing to lymph nodes, type I interferon, and immune suppression. Blood 112:4598-4608.

6. Stacey AR, Norris PJ, Qin L, Haygreen EA, Taylor E, Heitman J, Lebedeva M, DeCamp A, Li D, Grove D, Self SG, Borrow P. 2009. Induction of a striking systemic cytokine cascade prior to peak viremia in acute human immunodeficiency virus type 1 infection, in contrast to more modest and delayed responses in acute hepatitis $\mathrm{B}$ and $\mathrm{C}$ virus infections. J. Virol. 83:3719-3733.

7. Harris LD, Tabb B, Sodora DL, Paiardini M, Klatt NR, Douek DC, Silvestri G, Muller-Trutwin M, Vasile-Pandrea I, Apetrei C, Hirsch V, Lifson J, Brenchley JM, Estes JD. 2010. Downregulation of robust acute type I interferon responses distinguishes nonpathogenic simian immunodeficiency virus (SIV) infection of natural hosts from pathogenic SIV infection of rhesus macaques. J. Virol. 84:7886-7891.

8. Malim MH, Bieniasz PD. 2012. HIV restriction factors and mechanisms of evasion. Cold Spring Harb. Perspect. Med. 2:a006940. doi:10.1101 /cshperspect.a006940.

9. Neil S, Bieniasz P. 2009. Human immunodeficiency virus, restriction factors, and interferon. J. Interferon Cytokine Res. 29:569-580.

10. Le Tortorec A, Neil SJ. 2009. Antagonism to and intracellular sequestration of human tetherin by the human immunodeficiency virus type 2 envelope glycoprotein. J. Virol. 83:11966-11978.

11. Neil SJ, Zang T, Bieniasz PD. 2008. Tetherin inhibits retrovirus release and is antagonized by HIV-1 Vpu. Nature 451:425-430.

12. Van Damme N, Goff D, Katsura C, Jorgenson RL, Mitchell R, Johnson MC, Stephens EB, Guatelli J. 2008. The interferon-induced protein BST-2 restricts HIV-1 release and is downregulated from the cell surface by the viral Vpu protein. Cell Host Microbe 3:245-252.

13. Zhang F, Wilson SJ, Landford WC, Virgen B, Gregory D, Johnson MC, Munch J, Kirchhoff F, Bieniasz PD, Hatziioannou T. 2009. Nef proteins from simian immunodeficiency viruses are tetherin antagonists. Cell Host Microbe 6:54-67.

14. Jia B, Serra-Moreno R, Neidermyer W, Rahmberg A, Mackey J, Fofana IB, Johnson WE, Westmoreland S, Evans DT. 2009. Species-specific activity of SIV Nef and HIV-1 Vpu in overcoming restriction by tetherin/ BST2. PLoS Pathog. 5:e1000429. doi:10.1371/journal.ppat.1000429.

15. Ambrose Z, KewalRamani VN, Bieniasz PD, Hatziioannou T. 2007. HIV/AIDS: in search of an animal model. Trends Biotechnol. 25:333-337.

16. Stremlau M, Owens CM, Perron MJ, Kiessling M, Autissier P, Sodroski J. 2004. The cytoplasmic body component TRIM5alpha restricts HIV-1 infection in Old World monkeys. Nature 427:848-853.

17. Mariani R, Chen D, Schrofelbauer B, Navarro F, Konig R, Bollman B, Munk C, Nymark-McMahon H, Landau NR. 2003. Species-specific exclusion of APOBEC3G from HIV-1 virions by Vif. Cell 114:21-31.

18. Sheehy AM, Gaddis NC, Choi JD, Malim MH. 2002. Isolation of a human gene that inhibits HIV-1 infection and is suppressed by the viral Vif protein. Nature 418:646-650.

19. Virgen CA, Hatziioannou T. 2007. Antiretroviral activity and Vif sensitivity of rhesus macaque APOBEC3 proteins. J. Virol. 81:13932-13937.

20. Hatziioannou T, Princiotta M, Piatak M, Jr, Yuan F, Zhang F, Lifson JD, Bieniasz PD. 2006. Generation of simian-tropic HIV-1 by restriction factor evasion. Science 314:95. doi:10.1126/science.1130994.

21. Brennan G, Kozyrev Y, Hu SL. 2008. TRIMCyp expression in Old World primates Macaca nemestrina and Macaca fascicularis. Proc. Natl. Acad. Sci. U. S. A. 105:3569-3574.

22. Liao CH, Kuang YQ, Liu HL, Zheng YT, Su B. 2007. A novel fusion gene, TRIM5-cyclophilin A, in the pig-tailed macaque determines its susceptibility to HIV-1 infection. AIDS 21(Suppl. 8):S19-S26.

23. Virgen CA, Kratovac Z, Bieniasz PD, Hatziioannou T. 2008. Independent genesis of chimeric TRIM5-cyclophilin proteins in two primate species. Proc. Natl. Acad. Sci. U. S. A. 105:3563-3568.

24. Hatziioannou T, Ambrose Z, Chung NP, Piatak M, Jr, Yuan F, Trubey CM, Coalter V, Kiser R, Schneider D, Smedley J, Pung R, Gathuka M, Estes JD, Veazey RS, KewalRamani VN, Lifson JD, Bieniasz PD. 2009. A macaque model of HIV-1 infection. Proc. Natl. Acad. Sci. U. S. A. 106: 4425-4429.

25. Sunseri N, O'Brien M, Bhardwaj N, Landau NR. 2011. Human immunodeficiency virus type 1 modified to package simian immunodeficiency virus Vpx efficiently infects macrophages and dendritic cells. J. Virol. 85: 6263-6274.

26. Kestler H, Kodama T, Ringler D, Marthas M, Pedersen N, Lackner A, Regier D, Sehgal P, Daniel M, King N, et al. 1990. Induction of AIDS in 
rhesus monkeys by molecularly cloned simian immunodeficiency virus. Science 248:1109-1112.

27. Kimata JT, Mozaffarian A, Overbaugh J. 1998. A lymph node-derived cytopathic simian immunodeficiency virus Mne variant replicates in nonstimulated peripheral blood mononuclear cells. J. Virol. 72:245-256.

28. Zhang YJ, Hatziioannou T, Zang T, Braaten D, Luban J, Goff SP, Bieniasz PD. 2002. Envelope-dependent, cyclophilin-independent effects of glycosaminoglycans on human immunodeficiency virus type 1 attachment and infection. J. Virol. 76:6332-6343.

29. McNatt MW, Zang T, Hatziioannou T, Bartlett M, Fofana IB, Johnson WE, Neil SJ, Bieniasz PD. 2009. Species-specific activity of HIV-1 Vpu and positive selection of tetherin transmembrane domain variants. PLoS Pathog. 5:e1000300. doi:10.1371/journal.ppat.1000300.

30. Keele BF, Giorgi EE, Salazar-Gonzalez JF, Decker JM, Pham KT, Salazar MG, Sun C, Grayson T, Wang S, Li H, Wei X, Jiang C, Kirchherr JL, Gao F, Anderson JA, Ping LH, Swanstrom R, Tomaras GD, Blattner WA, Goepfert PA, Kilby JM, Saag MS, Delwart EL, Busch MP, Cohen MS, Montefiori DC, Haynes BF, Gaschen B, Athreya GS, Lee HY, Wood N, Seoighe C, Perelson AS, Bhattacharya T, Korber BT, Hahn BH, Shaw GM. 2008. Identification and characterization of transmitted and early founder virus envelopes in primary HIV-1 infection. Proc. Natl. Acad. Sci. U. S. A. 105:7552-7557.

31. Keele BF, Jones JH, Terio KA, Estes JD, Rudicell RS, Wilson ML, Li Y, Learn GH, Beasley TM, Schumacher-Stankey J, Wroblewski E, Mosser A, Raphael J, Kamenya S, Lonsdorf EV, Travis DA, Mlengeya T, Kinsel MJ, Else JG, Silvestri G, Goodall J, Sharp PM, Shaw GM, Pusey AE, Hahn BH. 2009. Increased mortality and AIDS-like immunopathology in wild chimpanzees infected with SIVcpz. Nature 460:515-519.

32. Lee HY, Giorgi EE, Keele BF, Gaschen B, Athreya GS, Salazar-Gonzalez JF, Pham KT, Goepfert PA, Kilby JM, Saag MS, Delwart EL, Busch MP, Hahn BH, Shaw GM, Korber BT, Bhattacharya T, Perelson AS. 2009. Modeling sequence evolution in acute HIV-1 infection. J. Theor. Biol. 261:341-360.

33. Salazar-Gonzalez JF, Bailes E, Pham KT, Salazar MG, Guffey MB, Keele BF, Derdeyn CA, Farmer P, Hunter E, Allen S, Manigart O, Mulenga J, Anderson JA, Swanstrom R, Haynes BF, Athreya GS, Korber BT, Sharp PM, Shaw GM, Hahn BH. 2008. Deciphering human immunodeficiency virus type 1 transmission and early envelope diversification by singlegenome amplification and sequencing. J. Virol. 82:3952-3970.

34. Salazar-Gonzalez JF, Salazar MG, Keele BF, Learn GH, Giorgi EE, Li H, Decker JM, Wang S, Baalwa J, Kraus MH, Parrish NF, Shaw KS, Guffey MB, Bar KJ, Davis KL, Ochsenbauer-Jambor C, Kappes JC, Saag MS, Cohen MS, Mulenga J, Derdeyn CA, Allen S, Hunter E, Markowitz M, Hraber P, Perelson AS, Bhattacharya T, Haynes BF, Korber BT, Hahn BH, Shaw GM. 2009. Genetic identity, biological phenotype, and evolutionary pathways of transmitted/founder viruses in acute and early HIV-1 infection. J. Exp. Med. 206:1273-1289.

35. Gao F, Yue L, Robertson DL, Hill SC, Hui H, Biggar RJ, Neequaye AE, Whelan TM, Ho DD, Shaw GM, Sharp PM, Hahn BH. 1994. Genetic diversity of human immunodeficiency virus type 2: evidence for distinct sequence subtypes with differences in virus biology. J. Virol. 68:74337447.

36. Owen SM, Ellenberger D, Rayfield M, Wiktor S, Michel P, Grieco MH, Gao F, Hahn BH, Lal RB. 1998. Genetically divergent strains of human immunodeficiency virus type 2 use multiple coreceptors for viral entry. J. Virol. 72:5425-5432.

37. Chesebro B, Wehrly K, Nishio J, Perryman S. 1992. Macrophage-tropic human immunodeficiency virus isolates from different patients exhibit unusual V3 envelope sequence homogeneity in comparison with T-celltropic isolates: definition of critical amino acids involved in cell tropism. J. Virol. 66:6547-6554.

38. Kappes JC, Parkin JS, Conway JA, Kim J, Brouillette CG, Shaw GM, Hahn BH. 1993. Intracellular transport and virion incorporation of vpx requires $[\mathrm{sic}]$ interaction with other virus type-specific components. Virology 193:222-233.

39. Cavacini LA, Emes CL, Wisnewski AV, Power J, Lewis G, Montefiori D, Posner MR. 1998. Functional and molecular characterization of human monoclonal antibody reactive with the immunodominant region of HIV type 1 glycoprotein 41. AIDS Res. Hum. Retroviruses 14:1271-1280.

40. Miyagi E, Andrew AJ, Kao S, Strebel K. 2009. Vpu enhances HIV-1 virus release in the absence of Bst-2 cell surface down-modulation and intracellular depletion. Proc. Natl. Acad. Sci. U. S. A. 106:2868-2873.
41. Kratovac Z, Virgen CA, Bibollet-Ruche F, Hahn BH, Bieniasz PD, Hatziioannou T. 2008. Primate lentivirus capsid sensitivity to TRIM5 proteins. J. Virol. 82:6772-6777.

42. Sauter D, Schindler M, Specht A, Landford WN, Munch J, Kim KA, Votteler J, Schubert U, Bibollet-Ruche F, Keele BF, Takehisa J, Ogando Y, Ochsenbauer C, Kappes JC, Ayouba A, Peeters M, Learn GH, Shaw G, Sharp PM, Bieniasz P, Hahn BH, Hatziioannou T, Kirchhoff F. 2009. Tetherin-driven adaptation of Vpu and Nef function and the evolution of pandemic and nonpandemic HIV-1 strains. Cell Host Microbe 6:409421.

43. Dazza MC, Ekwalanga M, Nende M, Shamamba KB, Bitshi P, Paraskevis D, Saragosti S. 2005. Characterization of a novel $v p u$-harboring simian immunodeficiency virus from a Dent's Mona monkey (Cercopithecus mona denti). J. Virol. 79:8560-8571.

44. Courgnaud V, Salemi M, Pourrut X, Mpoudi-Ngole E, Abela B, Auzel P, Bibollet-Ruche F, Hahn B, Vandamme AM, Delaporte E, Peeters M. 2002. Characterization of a novel simian immunodeficiency virus with a vpu gene from greater spot-nosed monkeys (Cercopithecus nictitans) provides new insights into simian/human immunodeficiency virus phylogeny. J. Virol. 76:8298-8309.

45. Hrecka K, Hao C, Gierszewska M, Swanson SK, Kesik-Brodacka M, Srivastava S, Florens L, Washburn MP, Skowronski J. 2011. Vpx relieves inhibition of HIV-1 infection of macrophages mediated by the SAMHD1 protein. Nature 474:658-661.

46. Laguette N, Sobhian B, Casartelli N, Ringeard M, Chable-Bessia C, Segeral E, Yatim A, Emiliani S, Schwartz O, Benkirane M. 2011. SAMHD1 is the dendritic- and myeloid-cell-specific HIV-1 restriction factor counteracted by Vpx. Nature 474:654-657.

47. Goldstone DC, Ennis-Adeniran V, Hedden JJ, Groom HC, Rice GI, Christodoulou E, Walker PA, Kelly G, Haire LF, Yap MW, de Carvalho LP, Stoye JP, Crow YJ, Taylor IA, Webb M. 2011. HIV-1 restriction factor SAMHD1 is a deoxynucleoside triphosphate triphosphohydrolase. Nature 480:379-382.

48. Lahouassa H, Daddacha W, Hofmann H, Ayinde D, Logue EC, Dragin L, Bloch N, Maudet C, Bertrand M, Gramberg T, Pancino G, Priet S, Canard B, Laguette N, Benkirane M, Transy C, Landau NR, Kim B, Margottin-Goguet F. 2012. SAMHD1 restricts the replication of human immunodeficiency virus type 1 by depleting the intracellular pool of deoxynucleoside triphosphates. Nat. Immunol. 13:223-228.

49. Laguette N, Rahm N, Sobhian B, Chable-Bessia C, Munch J, Snoeck J, Sauter D, Switzer WM, Heneine W, Kirchhoff F, Delsuc F, Telenti A, Benkirane M. 2012. Evolutionary and functional analyses of the interaction between the myeloid restriction factor SAMHD1 and the lentiviral Vpx protein. Cell Host Microbe 11:205-217.

50. Lim ES, Fregoso OI, McCoy CO, Matsen FA, Malik HS, Emerman M. 2012. The ability of primate lentiviruses to degrade the monocyte restriction factor SAMHD1 preceded the birth of the viral accessory protein Vpx. Cell Host Microbe 11:194-204.

51. Accola MA, Bukovsky AA, Jones MS, Gottlinger HG. 1999. A conserved dileucine-containing motif in $\mathrm{p} 6$ (gag) governs the particle association of $\mathrm{Vpx}$ and $\mathrm{Vpr}$ of simian immunodeficiency viruses SIV(mac) and SIV(agm). J. Virol. 73:9992-9999.

52. Horton R, Spearman P, Ratner L. 1994. HIV-2 viral protein X association with the GAG p27 capsid protein. Virology 199:453-457.

53. Gao F, Yue L, White AT, Pappas PG, Barchue J, Hanson AP, Greene BM, Sharp PM, Shaw GM, Hahn BH. 1992. Human infection by genetically diverse SIVSM-related HIV-2 in West Africa. Nature 358:495-499.

54. Ho DD, Hartshorn KL, Rota TR, Andrews CA, Kaplan JC, Schooley RT, Hirsch MS. 1985. Recombinant human interferon alfa-A suppresses HTLV-III replication in vitro. Lancet 1 (8429):602-604.

55. Berger A, Sommer AF, Zwarg J, Hamdorf M, Welzel K, Esly N, Panitz S, Reuter A, Ramos I, Jatiani A, Mulder LC, Fernandez-Sesma A, Rutsch F, Simon V, Konig R, Flory E. SAMHD1-deficient CD14 ${ }^{+}$cells from individuals with Aicardi-Goutieres syndrome are highly susceptible to HIV-1 infection. PLoS Pathog. 7:e1002425. doi:10.1371/journal.ppat .1002425.

56. Zhang F, Hatziioannou T, Perez-Caballero D, Derse D, Bieniasz PD. 2006. Antiretroviral potential of human tripartite motif- 5 and related proteins. Virology 353:396-409.

57. Schoggins JW, Wilson SJ, Panis M, Murphy MY, Jones CT, Bieniasz P, Rice CM. 2011. A diverse range of gene products are effectors of the type I interferon antiviral response. Nature 472:481-485. 